\title{
Quantum Entanglement and the Issue of Selective Influences in Psychology: An Overview
}

\author{
Ehtibar N. Dzhafarov ${ }^{1}$ and Janne V. Kujala ${ }^{2}$ \\ ${ }^{1}$ Purdue University \\ ehtibar@purdue.edu \\ ${ }^{2}$ University of Jyväskylä \\ jvk@iki.fi
}

\begin{abstract}
Similar formalisms have been independently developed in psychology, to deal with the issue of selective influences (deciding which of several experimental manipulations selectively influences each of several, generally non-independent, response variables), and in quantum mechanics (QM), to deal with the EPR entanglement phenomena (deciding whether an EPR experiment allows for a "classical" account). The parallels between these problems are established by observing that any two noncommuting measurements in QM are mutually exclusive and can therefore be treated as analogs of different values of one and the same input. Both problems reduce to that of the existence of a jointly distributed system of random variables, one variable for every value of every input (in psychology) or every measurement on every particle involved (in an EPR experiment). We overview three classes of necessary conditions (some of them also sufficient under additional constraints) for the existence of such joint distributions.
\end{abstract}

Keywords: Bell-CHSH-Fine inequalities, cosphericity test, EPR paradigm, joint distribution criterion, linear feasibility test, non-commuting measurements, pseudo-quasi-metrics on random variables, quantum entanglement, selective influences.

\section{Introduction}

Given a set of inputs into a system and a set of stochastically non-independent outputs, what is the precise meaning and means of ascertaining that a given output is not influenced by a given input? This paper reviews the developments related to this question.

The problem can be illustrated on the following diagram of selective influences:

\begin{tabular}{|ccc|}
\hline$\alpha^{1}=\{w, x, y\}$ & $\alpha^{2}=\{x\}$ & $\alpha^{3}=\{w, z\}$ \\
$\downarrow$ & $\downarrow$ & $\downarrow$ \\
$A^{1}$ & $A^{2}$ & $A^{3}$
\end{tabular}


$A^{1}, A^{2}$, and $A^{3}$ here are random outputs, $w, x, y, z$ are inputs (usually referred to as external factors in psychology and as measurement settings in QM), and arrows indicate the relation "may influence": thus, the diagram does not say that $A^{2}$ is necessarily influenced by $x$, but rather that $A^{2}$ is not influenced by $w, y, z$. The diagram is shown in the canonical form, i.e., the inputs are redefined, $\{w, x, y\}$ into $\alpha^{1},\{x\}$ into $\alpha^{2}$, etc., so that each output $A^{i}$ may only be influenced by a single input $\alpha^{i}$ that may not influence other outputs. We say then, for brevity, that $\left(A^{1}, A^{2}, A^{3}\right)$ are selectively influenced by $\left(\alpha^{1}, \alpha^{2}, \alpha^{3}\right)$ and write this as

$$
\left(A^{1}, A^{2}, A^{3}\right) \hookleftarrow\left(\alpha^{1}, \alpha^{2}, \alpha^{3}\right) .
$$

Inputs $\left(\alpha^{1}, \alpha^{2}, \alpha^{3}\right)$ are treated as deterministic quantities, i.e., even if they are random variables, the joint distribution of the outputs is always conditioned on their specific values. Each input can have one of several values, and the joint distribution of $\left(A^{1}, A^{2}, A^{3}\right)$ is known for each allowable treatment, a combination of input values. Thus, if $w, x, y, z$ are all binary, then $\alpha^{1}, \alpha^{2}, \alpha^{3}$ may be viewed as inputs with 8,2 , and 4 values, respectively, but the number of allowable treatments cannot exceed $16<8 \times 2 \times 4$. It can be less than 16 because some of the combinations may be physically impossible or simply not used or observed.

As a motivating example, consider a double-detection experiment in which two stimuli, say brief flashes, are presented simultaneously (right-left) or in a succession (first-second), each on one of two levels of intensity. The observer is asked to state, for each of the two observation areas (i.e., locations or time intervals), whether it contains a flash (Yes/No). The results of such an experiment are statistical estimates of 16 probabilities

$$
p\left(A^{1}, A^{2} \mid \alpha^{1}, \alpha^{2}\right)=\operatorname{Pr}\left[A^{1}:\left\{\begin{array}{c}
Y e s \\
N o
\end{array}, A^{2}:\left\{\begin{array}{c}
Y e s \\
N o
\end{array} \mid \alpha^{1}:\left\{\begin{array}{c}
\alpha_{1}^{1} \\
\alpha_{2}^{1}
\end{array}, \alpha^{2}:\left\{\begin{array}{c}
\alpha_{1}^{2} \\
\alpha_{2}^{2}
\end{array}\right],\right.\right.\right.\right.
$$

where $\alpha^{i}(i=1,2)$ is the input representing the $i$ th observation area (with values $\alpha_{1}^{i}, \alpha_{2}^{i}$ ), and $A^{i}$ is the response (Yes or No) to the $i$ th observation area. Assume that $A^{1}$ and $A^{2}$ for a given $\left(\alpha_{i}^{1}, \alpha_{j}^{2}\right)$ are not independent (due to attention fluctuations, perceptual learning, fatigue, etc.) In what sense then can we say that $\left(A^{1}, A^{2}\right) \leftrightarrow\left(\alpha^{1}, \alpha^{2}\right)$, and by what means can we find out if this is true?

Many empirical situations have precisely the same formal structure. In QM, an example is provided by the Bohmian version of the EPR paradigm [3]: two subatomic particles are emitted from a common source in such a way that they retain highly correlated spins as they run away from each other. An experiment may consist, e.g., in measuring the spin of electron 1 along one of two axes, $\alpha_{1}^{1}$ or $\alpha_{2}^{1}$, and (in another location but simultaneously in some inertial frame of reference) measuring the spin of electron 2 along one of two axes, $\alpha_{1}^{2}$ or $\alpha_{2}^{2}$. The outcome of a measurement on electron $1, A^{1}$, is a random variable with two possible values, "up" or "down," and the same holds for $A^{2}$, outcome of a measurement on electron 2. The question here is: for $i=1,2$, can we say that $A^{i}$ may only depend on $\alpha^{i}$, even though $A^{1}$ and $A^{2}$ are not independent? What makes this situation formally identical with the double-detection example is that the measurements along different axes, $\alpha_{1}^{i}$ and $\alpha_{2}^{i}$, are noncommuting, i.e., they 
cannot be performed on the $i$ th particle simultaneously. This makes it possible to consider them (measurements performed, not to be confused with their recorded outcomes) as mutually exclusive values of input $\alpha^{i}$. The results of such an experiment are described by (3), with Yes/No interpreted as spin up/down. In the original EPR paradigm [14] the non-commuting measurements are those of momentum and location, each with a continuum of possible values. Our parallel with the issue of selective influences requires that the measurements of the momentum and of the location of a given particle be interpreted as mutually exclusive values of one and the same input, "(measurement of the) momentum-location of the particle." This may be less intuitive than the analogous interpretation of the spins along different axes.

The question of selective influences cannot generally be decided based on the marginal distributions of the outputs alone. The most important example here is the classical CHSH experiment [4] where the marginal distributions of $A^{1}$ and $A^{2}$ (in the case of two electrons) remain constant, with $\operatorname{Pr}[\operatorname{spin} u p]=1 / 2$. Examples from psychology are also readily available, especially if one adopts a copula view of the joint distributions. Thus, $\alpha^{1}$ and $\alpha^{2}$ may represent two stimuli presented in a succession (each having several values), and $A^{1}, A^{2}$ be response times quantiles. The marginal distributions then are always the same, unit-uniform.

\section{A Historical Note}

The issue of selective influences was introduced to psychology in Sternberg's influential paper [22], in the context of studying consecutive "stages" of information processing. Sternberg acknowledged that selective influences can hold even if the durations of the stages are not stochastically independent, but he lacked mathematical apparatus for dealing with this possibility. Townsend [24] proposed to formalize the notion of selectively influenced and stochastically interdependent random variables by the concept of "indirect nonselectiveness": the conditional distribution of the variable $A^{1}$ given any value $a^{2}$ of the variable $A^{2}$, depends on $\alpha^{1}$ only, and, by symmetry, the conditional distribution of $A^{2}$ at any $A^{1}=a^{1}$ depends on $\alpha^{2}$ only. Under the name of "conditionally selective influence" this notion was mathematically characterized and generalized in [5]. Thus, if all combinations of values of inputs $\alpha^{1}, \alpha^{2}$ are allowable and random outputs $A^{1}, A^{2}$ are discrete, the diagram $\left(A^{1}, A^{2}\right) \stackrel{\text { cond }}{\leftarrow}\left(a^{1}, a^{2}\right)$, where $\stackrel{\text { cond }}{\leftarrow}$ means "is conditionally selectively influenced," holds if and only if $\operatorname{Pr}\left[A^{1}=a^{1}, A^{2}=a^{2} \mid \alpha_{x}^{1}, \alpha_{y}^{2}\right]$ can be presented as

$$
f_{12}\left(a^{1}, a^{2}\right) f_{1}\left(a^{1}, \alpha_{x}^{1}\right) f_{2}\left(a^{2}, \alpha_{y}^{2}\right) f\left(\alpha_{x}^{1}, \alpha_{y}^{2}\right),
$$

for all values $\left(a^{1}, a^{2}\right)$ of $\left(A^{1}, A^{2}\right)$ at all treatments $\left(\alpha_{x}^{1}, \alpha_{y}^{2}\right)$. Conditional selectivity is a useful notion, but it is not a satisfactory formalization of the intuitive notion of selective influences. The reason is that $\left(A^{1}, A^{2}\right) \stackrel{\text { cond }}{\leftarrow}\left(a^{1}, a^{2}\right)$ can be shown [5] to violate the following obvious property of an acceptable definition: 
the marginal distributions of $A^{1}$ and $A^{2}$ do not depend on, respectively, $\alpha^{2}$ and $\alpha^{1}$ ("marginal selectivity" [25]).

A different approach to selective influences, reviewed below, is based on $[6,7,9,10,11,12,19]$. As it turns out ${ }^{1}$ this approach parallels the development in QM of the issue of whether an EPR experiment can have a "classical" explanation (in terms of non-contextual local variables). The Joint Distribution Criterion which is at the heart of this development (see below) was indirectly introduced in the celebrated work of Bell [2], and explicitly in [15,16,23].

\section{Basic Notions}

Aimed at providing a broad overview of concepts and results, the content of this paper partially overlaps with that of several previous publications, especially $[11,12,19]$.

Random variables are understood in the broadest sense, as measurable functions $X: V_{s} \rightarrow V$, with no restrictions on the sample spaces $\left(V_{s}, \Sigma_{s}, \mu_{s}\right)$ and the induced probability spaces (distributions) $(V, \Sigma, \mu)$. In particular, any set $X$ of jointly distributed random variables (functions on the same sample space) is a random variable, and its distribution $(V, \Sigma, \mu)$ is referred to as the joint distribution of its elements. We use symbol $\sim$ in the meaning of "has the same distribution as." A random variable in the narrow sense is a special case of a random entity, with $V$ a finite product of countable sets and intervals of reals, and $\Sigma$ the smallest sigma-algebra containing the corresponding product of power sets and Lebesgue sigma-algebras. Note that a vector of random variables in the narrow sense is a random variable in the narrow sense.

Consider an indexed set $\alpha=\left\{\alpha^{\lambda}: \lambda \in \Lambda\right\}$, with each $\alpha^{\lambda}$ being a set referred to as a (deterministic) input, with the elements of $\{\lambda\} \times \alpha^{\lambda}$ called input points. Input points therefore are pairs of the form $x=(\lambda, w)$, with $w \in \alpha^{\lambda}$, and should not be confused with input values $w$. A nonempty set $\Phi \subset \prod_{\lambda \in \Lambda} \alpha^{\lambda}$ is called a set of (allowable) treatments. A treatment therefore is a function $\phi: \Lambda \rightarrow \bigcup_{\lambda \in \Lambda} \alpha^{\lambda}$ such that $\phi(\lambda) \in \alpha^{\lambda}$ for any $\lambda \in \Lambda$.

Let there be a collection of sets of random variables $A_{\phi}^{\lambda}(\lambda \in \Lambda, \phi \in \Phi)$, referred to as (random) outputs, with distributions $\left(V^{\lambda}, \Sigma^{\lambda}, \mu_{\phi}^{\lambda}\right)$. Let

$$
A_{\phi}=\left\{A_{\phi}^{\lambda}: \lambda \in \Lambda\right\}, \phi \in \Phi,
$$

be a random variable with a known distribution (the joint distribution of all $A_{\phi}^{\lambda}$ in $A_{\phi}$ ) for every treatment $\phi \in \Phi$. We define

$$
A^{\lambda}=\left\{A_{\phi}^{\lambda}: \phi \in \Phi\right\}, \lambda \in \Lambda,
$$

with the understanding that $A^{\lambda}$ is not generally a random variable, i.e., $A_{\phi}^{\lambda}$ for different $\phi$ are not necessarily jointly distributed. The definition of the relation

$$
\left\{A^{\lambda}: \lambda \in \Lambda\right\} \leftrightarrow\left\{\alpha^{\lambda}: \lambda \in \Lambda\right\},
$$

\footnotetext{
1 This was first pointed out to us by Jerome Busemeyer (personal communication, November 2010), for which we remain deeply grateful.
} 
interpreted as "for each $\lambda \in \Lambda, A^{\lambda}$ may be influenced by $\alpha^{\lambda}$ only," can be given in three equivalent forms:

$\left(\mathrm{SI}_{1}\right)$ there are independent random variables $C,\left\{S^{\lambda}: \lambda \in \Lambda\right\}$, and functions

$$
\left\{R^{\lambda}\left(w, C, S^{\lambda}\right): w \in \alpha^{\lambda}, \lambda \in \Lambda\right\},
$$

such that, for any treatment $\phi \in \Phi$,

$$
\left\{R^{\lambda}\left(\phi(\lambda), C, S^{\lambda}\right): \lambda \in \Lambda\right\} \sim A_{\phi} ;
$$

$\left(\mathrm{SI}_{2}\right)$ there is a random variable $C$ and functions

$$
\left\{P^{\lambda}(w, C): w \in \alpha^{\lambda}, \lambda \in \Lambda\right\},
$$

such that, for any treatment $\phi \in \Phi$,

$$
\left\{P^{\lambda}(\phi(\lambda), C): \lambda \in \Lambda\right\} \sim A_{\phi} ;
$$

(JDC) there is a set of jointly distributed random variables

$$
H=\left\{H_{w}^{\lambda}: w \in \alpha^{\lambda}, \lambda \in \Lambda\right\}
$$

(one random variable for every value of every input), such that, for any treatment $\phi \in \Phi$,

$$
\left\{H_{\phi(\lambda)}^{\lambda}: \lambda \in \Lambda\right\} \sim A_{\phi} .
$$

The latter statement constitutes the Joint Distribution Criterion (JDC) for selective influences, and $H$ is called the JDC (indexed) set. The proof of the equivalence [10] obtains essentially by the definition of a joint distribution, which seems to have been overlooked in the earlier derivations $[15,16]$. If $\Lambda=\{1, \ldots, n\}$ and all outputs $A^{\lambda}$ are random variables in the narrow sense, then $C$ in $\mathrm{Sl}_{2}$ and $C, S^{1}, \ldots, S^{n}$ in $\mathrm{Sl}_{1}$ can also be chosen to be random variables in the narrow sense; moreover, their distribution functions can be chosen arbitrarily, provided they are continuous and strictly increasing on their domains, e.g., unit uniform [11].

Two important consequences of (7) are as follows:

1. (nestedness) any subset $\Lambda^{\prime}$ of $\Lambda,\left\{A^{\lambda}: \lambda \in \Lambda^{\prime}\right\} \hookleftarrow\left\{\alpha^{\lambda}: \lambda \in \Lambda^{\prime}\right\}$; in particular, $\left\{A^{\lambda}: \lambda \in \Lambda^{\prime}\right\}$ may not depend on inputs outside $\Lambda^{\prime}$ (complete marginal selectivity);

2. (invariance with respect to input-value-specific transformations) for any set of measurable functions $\left\{F_{w}^{\lambda}(a): w \in \alpha^{\lambda}, \lambda \in \Lambda, a \in V^{\lambda}\right\}$,

$$
\left(B^{\lambda}: \lambda \in \Lambda\right) \hookleftarrow\left\{\alpha^{\lambda}: \lambda \in \Lambda\right\}
$$

where $B^{\lambda}=\left\{B_{\phi}^{\lambda}: \phi \in \Phi\right\}$, and $B_{\phi}^{\lambda}=F_{\phi(\lambda)}^{\lambda}\left(A_{\phi}^{\lambda}\right)$. 
These properties should be viewed as desiderata for any reasonable definition of selective influences.

In $\mathrm{QM}, \mathrm{SI}_{1}$ corresponds to the existence of a "classical" probabilistic explanation. In psychology, statement $\mathrm{SI}_{1}$ combined with auxiliary assumptions was used in [8] and [20] to analyze the representability of same-different pairwise discrimination probabilities by means of Thurstonian-type models in which two stimuli being compared are mapped into random entities (distributed in some hypothetical space of mental images) that in turn are mapped (deterministically or probabilistically) into a response, "same" or "different." Statement $\mathrm{SI}_{1}$ was also used to analyze the response time distributions for parallel-serial networks of mental operations with selectively influenced components [13]. Note that the representation of the outputs $A^{\lambda}$ as functions of the corresponding inputs $\alpha^{i}$ and unobservable sources of randomness, $\mathrm{A}^{\lambda}$-specific $\left(S^{\lambda}\right)$ and common $(C)$, includes as special cases all conceivable generalizations and combinations of regression and factor analyses, with our term "input" corresponding to the traditional "regressor," and the term "source of randomness" to the factor-analytic "factor." This observation alone shows the potentially unlimited sphere of applicability of $\mathrm{SI}_{1}$.

Statement $\mathrm{SI}_{2}$ (corresponding in QM to "classical" determinsitic explanation) and JDC turn out to be more convenient in dealing with certain foundational probabilistic issues [9] and for the construction of the working tests (necessary conditions) for selective influences $[10,11,12,19]$. The tests are discussed below.

The following is a table of correspondences between the general terminology used in dealing with the issue of selective influences, and that of QM in dealing with EPR.

\begin{tabular}{|c|c|}
\hline Selective Probabilistic Causality (general) & Quantum Entanglement Problem \\
\hline \hline observed random output & $\begin{array}{c}\text { outcome of a given measurement } \\
\text { on a given particle }\end{array}$ \\
\hline input (factor) & $\begin{array}{c}\text { set of noncommuting measurements } \\
\text { on a given particle }\end{array}$ \\
\hline input value & $\begin{array}{c}\text { one of noncommuting measurements } \\
\text { on a given particle }\end{array}$ \\
\hline joint distribution criterion & joint distribution criterion \\
\hline diagram of selective influences & "classical" explanation \\
\hline representation in the form $\mathrm{SI}_{1}$ & probabilistic "classical" explanation \\
\hline representation in the form $\mathrm{SI}_{2}$ & deterministic "classical" explanation \\
\hline
\end{tabular}

\section{Tests for Selective Influences}

Let $H=\left\{H_{w}^{\lambda}: w \in \alpha^{\lambda}, \lambda \in \Lambda\right\}$ be a hypothetical JDC-set, i.e., a set satisfying (13) but not necessarily jointly distributed. Denoting

$$
\left\{H_{\phi(\lambda)}^{\lambda}: \lambda \in \Lambda\right\}=H_{\phi}, \phi \in \Phi,
$$


let $\mathcal{H}$ be a set of constraints imposed on possible distributions of $H_{\phi}$. For instance, $\mathcal{H}$ may be the requirement that all $H_{\phi}^{\lambda}$ be composed of Bernoulli variables, or multivariate-normally distributed.

A statement $S\left(H_{\phi_{1}}, \ldots, H_{\phi_{s}}\right)$, with $\phi_{1}, \ldots, \phi_{s} \in \Phi$, is called a test for the relation (7) under constraints $\mathcal{H}$, if

1. (observability) its truth value only depends on the distributions of $H_{\phi_{1}}, \ldots, H_{\phi_{s}}$;

2. (non-emptiness) it is not true for all possible distributions of $H_{\phi_{1}}, \ldots, H_{\phi_{s}}$ satisfying $\mathcal{H}$

3. (necessity) it is true if $H$ is jointly distributed.

If $S\left(H_{\phi_{1}}, \ldots, H_{\phi_{s}}\right)$ is false for all distributions of $H_{\phi_{1}}, \ldots, H_{\phi_{s}}$ satisfying $\mathcal{H}$ unless $H$ is jointly distributed, the test is called a criterion for (7). In the following we assume that $\mathcal{H}$ always includes the requirement of complete marginal selectivity: for any $\Lambda^{\prime} \subset \Lambda$, the joint distribution of $\left\{A_{\phi\left(\Lambda^{\prime}\right) \cup \phi\left(\Lambda-\Lambda^{\prime}\right)}^{\lambda}: \lambda \in \Lambda^{\prime}\right\}$ does not depend on $\phi\left(\Lambda-\Lambda^{\prime}\right)$. If this condition is violated, (7) is ruled out trivially.

\subsection{Pseudo-quasi-distance tests}

A function $d: H \times H \rightarrow \mathbb{R}$ is a pseudo-quasi-metric (p.q.-metric) on $H$ if, for any $H_{1}, H_{2}, H_{3} \in H$,

(i) $d\left(H_{1}, H_{2}\right)$ only depends on the joint distribution of $\left(H_{1}, H_{2}\right)$,

(ii) $d\left(H_{1}, H_{2}\right) \geq 0$,

(iii) $d\left(H_{1}, H_{1}\right)=0$,

(iv) $d\left(H_{1}, H_{3}\right) \leq d\left(H_{1}, H_{2}\right)+d\left(H_{2}, H_{3}\right)$.

The conventional pseudometrics (also called semimetrics) obtain by adding the property $d\left(H_{1}, H_{2}\right)=d\left(H_{2}, H_{1}\right)$; the conventional quasimetrics are obtained by adding the property $\operatorname{Pr}\left[H_{1}=H_{2}\right]<1 \Rightarrow d\left(H_{1}, H_{2}\right)>0$. A conventional metric is both a pseudometric and a quasimetric.

A sequence of input points

$$
x_{1}=\left(\lambda_{1}, w_{1}\right), \ldots, x_{l}=\left(\lambda_{l}, w_{l}\right),
$$

where $w_{i} \in \alpha^{\lambda_{i}}$ for $i=1, \ldots, l \geq 3$, is called treatment-realizable if there are treatments $\phi^{1}, \ldots, \phi^{l} \in \Phi$ (not necessarily pairwise distinct), such that

$$
\left\{x_{1}, x_{l}\right\} \subset \phi^{1} \text { and }\left\{x_{i-1}, x_{i}\right\} \subset \phi^{i} \text { for } i=2, \ldots, l .
$$

If a JDC-set $H$ exists, then for any p.q.-metric $d$ on $H$ we should have

$$
d\left(H_{w_{1}}^{\lambda_{1}}, H_{w_{l}}^{\lambda_{l}}\right)=d\left(A_{\phi^{1}}^{\lambda_{1}}, A_{\phi^{1}}^{\lambda_{l}}\right)
$$

and

$$
d\left(H_{w_{i-1}}^{\lambda_{i-1}}, H_{w_{i}}^{\lambda_{i}}\right)=d\left(A_{\phi^{i}}^{\lambda_{i-1}}, A_{\phi^{i}}^{\lambda_{i}}\right), i=2, \ldots, l,
$$

whence

$$
d\left(A_{\phi^{1}}^{\lambda_{1}}, A_{\phi^{1}}^{\lambda_{l}}\right) \leq \sum_{i=2}^{l} d\left(A_{\phi^{i}}^{\lambda_{i-1}}, A_{\phi^{i}}^{\lambda_{i}}\right)
$$


This chain inequality constitutes a p.q.-metric test for selective influences. If this inequality is found not to hold for at least one treatment-realizable sequence of input points, selectivity (7) is ruled out [12].

It turns out that one needs to check the chain inequality only for irreducible treatment-realizable sequences $x_{1}, \ldots, x_{l}$, i.e., those with $x_{1} \neq x_{l}$ and with the property that the only subsequences $\left\{x_{i_{1}}, \ldots, x_{i_{k}}\right\}$ with $k>1$ that are subsets of treatments are pairs $\left\{x_{1}, x_{l}\right\}$ and $\left\{x_{i-1}, x_{i}\right\}$, for $i=2, \ldots, l$. Inequality (20) is satisfied for all treatment-realizable sequences if and only if it holds for all irreducible sequences [12]. The situation is even simpler if $\Phi=\prod_{\lambda \in \Lambda} W^{\lambda}$ (all logically possible treatments are allowable). Then (20) is satisfied for all treatment-realizable sequences if and only if this inequality holds for all tetradic sequences of the form $x, y, s, t$, with $x, s \in\left\{\lambda_{1}\right\} \times \alpha^{\lambda_{1}}, y, t \in\left\{\lambda_{2}\right\} \times \alpha^{\lambda_{2}}, x \neq s$, $y \neq t, \lambda_{1} \neq \lambda_{2}[10]$.

Order-distances constitute a special class of p.q.-metrics, defined as follows. Let the distribution of $H_{w}^{\lambda} \in H$ be $\left(V^{\lambda}, \Sigma^{\lambda}, \mu_{w}^{\lambda}\right)$. Let

$$
R \subset \bigcup_{\left(\lambda_{1}, \lambda_{2}\right) \in \Lambda \times \Lambda} V^{\lambda_{1}} \times V^{\lambda_{2}},
$$

and let us write $a \preceq b$ for $(a, b) \in R$. Let $R$ be a total order (transitive, reflexive, and connected). We assume that for any $\left(\lambda_{1}, \lambda_{2}\right) \in \Lambda \times \Lambda, \operatorname{Pr}\left[H_{w_{1}}^{\lambda_{1}} \preceq H_{w_{2}}^{\lambda_{2}}\right]$ is well-defined, i.e., $\left\{(a, b): a \in V^{\lambda_{1}}, b \in V^{\lambda_{2}}, a \preceq b\right\}$ belongs to the product sigmaalgebra over $\Sigma^{\lambda_{1}}$ and $\Sigma^{\lambda_{2}}$. Then the function

$$
\mathrm{D}\left(H_{w_{1}}^{\lambda_{1}}, H_{w_{2}}^{\lambda_{2}}\right)=\operatorname{Pr}\left[H_{w_{1}}^{\lambda_{1}} \prec H_{w_{2}}^{\lambda_{2}}\right]
$$

where $\prec$ is the strict order induced by $\preceq$, is well-defined, and it is a p.q.-metric on $H$, called order-distance [12].

As a simple example, consider the results of a CHSH type experiment with two spin axes per each of two entangled 1/2-spin particles. Enumerate the spin axes 1,2 for either particle, enumerate the two outcomes (up and down) of each measurement 1,2 for particle 1 and $1^{\prime}, 2^{\prime}$ for particle 2 , and denote

$$
\operatorname{Pr}\left[H_{i}^{1}=k, H_{j}^{2}=l^{\prime}\right]=\operatorname{Pr}\left[A_{(i, j)}^{1}=k, A_{(i, j)}^{2}=l^{\prime}\right]=p_{k l \mid i j},
$$

where $i, j, k, l \in\{1,2\}$. Define the order-distance $\mathrm{D}_{1}$ by putting $1 \simeq 1^{\prime} \prec 2 \simeq 2^{\prime}$, where $\simeq$ is equivalence induced by $\preceq$. We have then the chain inequality

$$
\begin{aligned}
& p_{12 \mid 12}=\mathrm{D}_{1}\left(H_{1}^{1}, H_{2}^{2}\right) \\
& \quad \leq \mathrm{D}_{1}\left(H_{1}^{1}, H_{1}^{2}\right)+\mathrm{D}_{1}\left(H_{1}^{2}, H_{2}^{1}\right)+\mathrm{D}_{1}\left(H_{2}^{1}, H_{2}^{2}\right)=p_{12 \mid 11}+p_{21 \mid 21}+p_{12 \mid 22} .
\end{aligned}
$$

Consider next a similar inequality for the order-distance $\mathrm{D}_{2}$ defined by $1 \simeq 2^{\prime} \prec$ $2 \simeq 1^{\prime}$ :

$$
\begin{aligned}
& p_{11 \mid 12}=\mathrm{D}_{2}\left(H_{1}^{1}, H_{2}^{2}\right) \\
& \quad \leq \mathrm{D}_{2}\left(H_{1}^{1}, H_{1}^{2}\right)+\mathrm{D}_{2}\left(H_{1}^{2}, H_{2}^{1}\right)+\mathrm{D}_{2}\left(H_{2}^{1}, H_{2}^{2}\right)=p_{11 \mid 11}+p_{22 \mid 21}+p_{11 \mid 22} .
\end{aligned}
$$


By simple algebra, denoting

$$
\operatorname{Pr}\left[H_{i}^{1}=k\right]=p_{k \cdot \mid i \cdot}, \operatorname{Pr}\left[H_{j}^{2}=l^{\prime}\right]=p_{\cdot l \mid \cdot j},
$$

the conjunction of (24) and (25) can be shown to be equivalent to

$$
-1 \leq p_{11 \mid 11}+p_{11 \mid 21}+p_{11 \mid 22}-p_{11 \mid 12}-p_{1 \cdot \mid 2 \cdot}-p_{\cdot 1 \mid \cdot 1} \leq 0 .
$$

One derives analogously

$$
\begin{aligned}
& -1 \leq p_{11 \mid 12}+p_{11 \mid 22}+p_{11 \mid 21}-p_{11 \mid 11}-p_{1 \cdot \mid 2 \cdot}-p_{\cdot 1 \mid \cdot 2} \leq 0, \\
& -1 \leq p_{11 \mid 21}+p_{11 \mid 11}+p_{11 \mid 12}-p_{11 \mid 22}-p_{1 \cdot \mid 1 \cdot}-p_{\cdot 1 \mid \cdot 1} \leq 0, \\
& -1 \leq p_{11 \mid 22}+p_{11 \mid 12}+p_{11 \mid 11}-p_{11 \mid 21}-p_{1 \cdot \mid 1 \cdot}-p_{\cdot 1 \mid \cdot 2} \leq 0 .
\end{aligned}
$$

The four double-inequalities (27)-(28) can be referred to as the Bell-CHSHFine inequalities $[15,16]$, necessary and sufficient conditions for the CHSH type experiment to have a "classical" explanation.

\subsection{Cosphericity Tests}

Let the outputs $A_{\phi}^{\lambda}$ all be random variables in the narrow sense. Denote, for any distinct $\lambda_{1}, \lambda_{2} \in \Lambda$ and any $\phi \in \Phi$ with $\phi\left(\lambda_{1}\right)=w_{1}$ and $\phi\left(\lambda_{2}\right)=w_{2}$,

$$
\operatorname{Cor}\left[H_{w_{1}}^{\lambda_{1}}, H_{w_{2}}^{\lambda_{2}}\right]=\operatorname{Cor}\left[A_{\phi}^{\lambda_{1}}, A_{\phi}^{\lambda_{2}}\right]=\rho_{w_{1} w_{2}}^{\lambda_{1} \lambda_{2}},
$$

where Cor designates correlation. Let $\phi_{1}, \phi_{2}, \phi_{3}, \phi_{4} \in \Phi$ be any treatments with

$$
\begin{aligned}
& \phi_{1}\left(\lambda_{1}\right)=\phi_{2}\left(\lambda_{1}\right)=w_{1} ; \phi_{1}\left(\lambda_{2}\right)=\phi_{3}\left(\lambda_{2}\right)=w_{2} \\
& \phi_{4}\left(\lambda_{1}\right)=\phi_{2}\left(\lambda_{1}\right)=w_{1}^{\prime} ; \phi_{4}\left(\lambda_{2}\right)=\phi_{3}\left(\lambda_{2}\right)=w_{2}^{\prime} .
\end{aligned}
$$

Then, as shown in [19], if the components of $H$ are jointly distributed,

$$
\begin{aligned}
& \left|\rho_{w_{1} w_{2}}^{\lambda_{1} \lambda_{2}} \rho_{w_{1} w_{2}^{\prime}}^{\lambda_{1} \lambda_{2}}-\rho_{w_{1}^{\prime} w_{2}}^{\lambda_{1} \lambda_{2}} \rho_{w_{1}^{\prime} w_{2}^{\prime}}^{\lambda_{1} \lambda_{2}}\right| \\
& \quad \leq \sqrt{1-\left(\rho_{w_{1} w_{2}}^{\lambda_{1} \lambda_{2}}\right)^{2}} \sqrt{1-\left(\rho_{w_{1} w_{2}^{\prime}}^{\lambda_{1} \lambda_{2}}\right)^{2}}+\sqrt{1-\left(\rho_{w_{1}^{\prime} w_{2}}^{\lambda_{1} \lambda_{2}}\right)^{2}} \sqrt{1-\left(\rho_{w_{1}^{\prime} w_{2}^{\prime}}^{\lambda_{1} \lambda_{2}}\right)^{2}},
\end{aligned}
$$

This is the cosphericity test for (7), called so because geometrically (31) describes the possibility to place four points $\left(w_{1}, w_{2}, w_{1}^{\prime}, w_{2}^{\prime}\right)$ on a unit sphere in $3 \mathrm{D}$ Euclidean space so that the angles between the corresponding radius-vectors have cosines equal to the correlations. Note that an outcome of this test does not allow to predict the outcome of the same test applied to nonlinearly input-valuespecifically transformed random variables. Due to (14), this creates a multitude of cosphericity tests for one and the same initial set of outputs $A_{\phi}^{\lambda}$.

In the all-important for behavioral sciences $2 \times 2$ factorial design $(\Lambda=\{1,2\}$, each input is binary, and $\Phi$ consists of all four possible treatments), the cosphericity test is a criterion for $\left(A^{1}, A^{2}\right) \hookleftarrow\left(\alpha^{1}, \alpha^{2}\right)$ if (perhaps following some inputvalue-specific transformation) the outputs are bivariate normally distributed for all four treatments [19]. 


\subsection{Linear Feasibility Test}

The Linear Feasibility Test (LFT) is a criterion for selective influences in all situations involving finite sets of inputs/outputs, $\Lambda=\{1, \ldots, n\}$, with the $i$ th input and $i$ th output having finite sets of values, $\left\{1, \ldots, k_{i}\right\}$ and $\left\{1, \ldots, m_{i}\right\}$, respectively [11]. In other situations LFT can be used as a necessary condition because every set of possible values can be discretized. The distributions of $H_{\phi}=\left(H_{j_{1}}^{1}, \ldots, H_{j_{n}}^{n}\right)$ are represented by probabilities

$$
\operatorname{Pr}\left[H_{j_{1}}^{1}=a_{1}, \ldots, H_{j_{n}}^{n}=a_{n}\right]=\operatorname{Pr}\left[A_{\phi}^{1}=a_{1}, \ldots, A_{\phi}^{n}=a_{n}\right],
$$

with $\phi=\left(j_{1}, \ldots, j_{n}\right) \in \Phi$ and

$$
\left(a_{1}, \ldots, a_{n}\right) \in\left\{1, \ldots, m_{1}\right\} \times \cdots \times\left\{1, \ldots, m_{n}\right\} .
$$

We consider this probability the $\left[\left(a_{1}, \ldots, a_{n}\right),\left(j_{1}, \ldots, j_{n}\right)\right]$ th component of the $m_{1} \cdots m_{n} t$-vector $P$ (with $t$ denoting the number of treatments in $\Phi$ ). The joint distribution of $H$ in JDC, if it exists, is represented by probabilities

$$
\operatorname{Pr}\left[H_{1}^{1}=h_{1}^{1} \ldots, H_{k_{1}}^{1}=h_{k_{1}}^{1}, \ldots, H_{1}^{n}=h_{1}^{n}, \ldots, H_{k_{n}}^{n}=h_{k_{n}}^{n}\right],
$$

with

$$
\left(h_{1}^{1}, \ldots, h_{k_{1}}^{1}, \ldots, h_{1}^{n}, \ldots, h_{k_{n}}^{n}\right) \in\left\{1, \ldots, m_{1}\right\}^{k_{1}} \times \ldots \times\left\{1, \ldots, m_{n}\right\}^{k_{n}} .
$$

We consider this probability the $\left(h_{1}^{1}, \ldots, h_{k_{1}}^{1}, \ldots, h_{1}^{n}, \ldots, h_{k_{n}}^{n}\right)$ th component of the $\left(m_{1}\right)^{k_{1}} \cdots\left(m_{n}\right)^{k_{n}}$-vector $Q$. Consider now the Boolean matrix $M$ with rows corresponding to components of $P$ and columns to components of $Q$ : let $M(r, c)=$ 1 if and only if

1. row $r$ corresponds to the $\left[\left(j_{1}, \ldots, j_{n}\right),\left(a_{1}, \ldots, a_{n}\right)\right]$ th component of $P$,

2. column $c$ to the $\left(h_{1}^{1}, \ldots, h_{k_{1}}^{1}, \ldots, h_{1}^{n}, \ldots, h_{k_{n}}^{n}\right)$ th component of $Q$, and

3. $h_{j_{1}}^{1}=a_{1}, \ldots, h_{j_{n}}^{n}=a_{n}$.

Clearly, the vector $Q$ exists if and only if the system

$$
M Q=P, Q \geq 0
$$

has a solution (is feasible). This is a linear programming task in the standard form (with a constant objective function). Let $\mathcal{L}(P)$ be a Boolean function equal to 1 if and only if this system is feasible. $\mathcal{L}(P)$ is known to be computable, its time complexity being polynomial [18].

The potential of JDC to lead to LFT and provide an ultimate criterion for the Bohmian entanglement problem has not been utilized in quantum physics until relatively recently, when LFT was proposed in [26,27] and [1]. But the essence of the idea can be found in [21]. Given a set of numerical (experimentally estimated or theoretical) probabilities, computing $\mathcal{L}(P)$ is always preferable to dealing with explicit inequalities as their number becomes very large even for moderate-size vectors $P$. The classical Bell-CHSH-Fine inequalities (27)-(28) for 
$n=2, k_{1}=k_{2}=2, m_{1}=m_{2}=2$ (assuming that the marginal selectivity equalities hold) number just 8 , but already for $n=2, k_{1}=k_{2}=2$ with $m_{1}=$ $m_{2}=3$ (describing, e.g., an EPR experiment with two spin-1 particles, or two spin- $1 / 2$ ones and inefficient detectors), our computations yield 1080 inequalitiies equivalent to $\mathcal{L}(P)=1$. For $n=3, k_{1}=k_{2}=k_{3}=2$ and $m_{1}=m_{2}=m_{3}=$ 2 , corresponding to the GHZ paradigm [17] with three spin- $1 / 2$ particles, this number is 53792. Lists of such inequalities can be derived "mechanically" from the format of matrix $M$ using well-known facet enumeration algorithms (see, e.g., program lrs at http://cgm.cs.mcgill.ca/ avis/C/lrs.html). Once such a system of inequalities $S$ is derived, one can use it to prove necessity (or sufficiency) of any other system $S^{\prime}$ by showing, with the aid of a linear programming algorithm, that $S^{\prime}$ is redundant when added to $S$ (respectively, $S$ is redundant when added to $\left.S^{\prime}\right)$.

Acknowledgments. This research has been supported by the NSF grant SES1155956 to Purdue University and the Academy of Finland grant 121855 to University of Jyväskylä.

\section{References}

1. Basoalto, R.M., Percival, I.C.: BellTest and CHSH experiments with more than two settings. Journal of Physics A: Mathematical \& General 36, 7411-7423 (2003)

2. Bell, J.: On the Einstein-Podolsky-Rosen paradox. Physics 1, 195-200 (1964)

3. Bohm, D., Aharonov, Y.: Discussion of Experimental Proof for the Paradox of Einstein, Rosen and Podolski. Physical Review 108, 1070-1076 (1957)

4. Clauser, J.F., Horne, M.A., Shimony, A., Holt, R.A.: Proposed experiment to test local hidden-variable theories. Physical Review Letters 23, 880-884 (1969)

5. Dzhafarov, E.N.: Conditionally selective dependence of random variables on external factors. Journal of Mathematical Psychology 43, 123-157 (1999)

6. Dzhafarov, E.N.: Unconditionally selective dependence of random variables on external factors. Journal of Mathematical Psychology 45, 421-451 (2001)

7. Dzhafarov, E.N.: Selective influence through conditional independence. Psychometrika 68, 7-26 (2003)

8. Dzhafarov, E.N.: Thurstonian-type representations for "same-different" discriminations: Probabilistic decisions and interdependent images. Journal of Mathematical Psychology 47, 229-243 (2003) [see Dzhafarov, E.N.: Corrigendum to "Thurstoniantype representations for 'same-different' discriminations: Probabilistic decisions and interdependent images." Journal of Mathematical Psychology 50, 511 (2006)]

9. Dzhafarov, E.N., Gluhovsky, I.: Notes on selective influence, probabilistic causality, and probabilistic dimensionality. Journal of Mathematical Psychology 50, 390-401 (2006)

10. Dzhafarov, E.N., Kujala, J.V.: The Joint Distribution Criterion and the Distance Tests for Selective Probabilistic Causality. Frontiers in Quantitative Psychology and Measurement 1:151 doi: 10.3389/fpsyg.2010.0015 (2010)

11. Dzhafarov, E.N., Kujala, J.V.: Selectivity in probabilistic causality: Where psychology runs into quantum physics. Journal of Mathematical Psychology 56, 54-63 (2012) 
12. Dzhafarov, E.N., Kujala, J.V.: Order-distance and other metric-like functions on jointly distributed random variables. Proceedings of the American Mathematical Society (in press as of 2011)

13. Dzhafarov, E.N., Schweickert, R., Sung, K.: Mental architectures with selectively influenced but stochastically interdependent components. Journal of Mathematical Psychology 48, 51-64 (2004)

14. Einstein, A., Podolsky, B., Rosen N.: Can Quantum-Mechanical Description of Physical Reality be Considered Complete? Physical Review 47, 777-780 (1935)

15. Fine, A.: Joint distributions, quantum correlations, and commuting observables. Journal of Mathematical Physics 23, 1306-1310 (1982)

16. Fine, A.: Hidden variables, joint probability, and the Bell inequalities. Physical Review Letters 48, 291-295 (1982)

17. Greenberger, D.M., Horne, M.A., Zeilinger, A.: Going beyond Bell's theorem. In: M. Kafatos (ed.) Bell's Theorem, Quantum Theory and Conceptions of the Universe, pp. 69-72. Kluwer, Dordrecht (1989)

18. Karmarkar, N.: A new polynomial-time algorithm for linear programming. Combinatorica 4, 373-395 (1984)

19. Kujala, J.V., Dzhafarov, E.N.: Testing for selectivity in the dependence of random variables on external factors. Journal of Mathematical Psychology 52, 128-144 (2008)

20. Kujala, J.V., Dzhafarov, E.N.: Regular Minimality and Thurstonian-type modeling. Journal of Mathematical Psychology 53, 486-501 (2009)

21. Pitowski, I.: Quantum Probability - Quantum Logic. Springer, Berlin (1989)

22. Sternberg, S.: The discovery of processing stages: Extensions of donders' method. Acta Psychologica 30, 276-315 (1969)

23. Suppes, P., Zanotti, M.: When are probabilistic explanations possible? Synthese 48, 191-199 (1981)

24. Townsend, J. T.: Uncovering mental processes with factorial experiments. Journal of Mathematical Psychology 28, 363-400 (1984)

25. Townsend, J.T., Schweickert, R.: Toward the trichotomy method of reaction times: Laying the foundation of stochastic mental networks. Journal of Mathematical Psychology 33, 309-327 (1989)

26. Werner, R.F., Wolf, M.M.: All multipartite Bell correlation inequalities for two dichotomic observables per site. arXiv:quant-ph/0102024v1 (2001)

27. Werner, R.F., Wolf, M.M.: Bell inequalities and entanglement. arXiv:quant-ph/ 0107093 v2 (2001) 